\title{
Une brève histoire de la culture technique européenne et de sa relation à l'innovation
}

\author{
A brief history of European technical culture and its relationship to \\ innovation
}

\author{
Anne-Françoise Garçon ${ }^{1}$ \\ ${ }^{1}$ Centre d'Histoire des Techniques, Université Paris 1 Panthéon-Sorbonne, afgarcon@univ-paris1.fr
}

\begin{abstract}
RÉSUMÉ. Toute culture technique se compose d'éléments de langage, vocabulaire et/ou technolecte, et d'un faisceau de pratiques sociales qui détermine un habitus technique. Et toute communauté de métier possède sa culture technique. Les formes en diffèrent toutefois selon le régime d'appropriation de la technique autour duquel chaque communauté se structure : oralité et symbolisme technique pour le régime de la pratique, traité technique et symbolisme mathématique pour le régime de la technique, conception assistée par ordinateur et analyse systémique pour le régime de la technologie. Historiquement, l'objectivation de la notion de progrès, quoique présente dans le régime de la pratique, s'est effectuée au XVle siècle, alors que le régime de la technique prenait une ampleur jusque-là inégalée. Mais sans déboucher sur une culture d'innovation. Celle-ci s'instaura en Europe, à la fin du XIXe siècle, avec la seconde industrialisation, à partir de cette culture " tableau de bord » (O. Barfield), mélange d'idéologie scientiste et progressiste qui fonde la co-activité dans les sociétés industrielles.

ABSTRACT. All technical culture consists of language elements, vocabulary and / or "technolecte", and a plethora of social practices that define a technical habitus. Each trade and profession has its own technical culture. The forms differ from one another according to the three major systems of appropriation of the techniques on which each trade is founded upon: orality and technical symbolism for the pragmatic system, technical treatise and mathematical symbolism for the technical system, computer-assistance and systemic analysis for the technological system. Historically, the objectivization of the notion of progress, although present in the pragmatic system, took place in the sixteenth century when the technical system became the predominant pattern. But without leading to a culture of innovation which appeared in Europe only at the end of the nineteenth century, with the second industrialization, and built on the basis of the "dashboard culture"; $(\mathrm{O}$. Barfield), shared by all professions, a blend of scientistic and progressive ideology, which founded co-activity in industrial societies.
\end{abstract}

MOTS-CLÉS. Culture technique, invention, innovation, régime d'appropriation de la technique, grammatisation, énoncés techniques, technologie, théorie $\mathrm{C} / \mathrm{K}$, génie industriel.

KEYWORDS. Technical culture, operative knowledge, dashboard culture, engineering studies, $\mathrm{C} / \mathrm{K}$ theory, innovation, invention, technological writings.

Les deux approches historiques de l'innovation et de la technique ont en commun de faire apparaître un différentiel de temporalités entre les deux historicités, du fait et de la notion ${ }^{1}$. Inventer, faire du nouveau est une constante de l'histoire humaine. "Le novateur est un chef qui n'a pas à agir («prattein »), il gouverne («archein ») ceux qui sont capables d'exécuter », écrivait Platon dans Le Politique [ARE 94]. Par contre, mettre à distance cette capacité à faire du neuf, objectiver l'innovation pour en faire une notion et construire autour d'elle une culture dédiée, n'est pas allé de soi. Il en va de même du fait technique. La technique, matérielle et/ou immatérielle, accompagne l'histoire des sociétés humaine depuis la nuit des temps. Pour autant, la technique n'est pas nécessairement objectivée y compris de nos jours. L'histoire des régimes d'appropriation du fait technique donne à voir l'émergence tardive d'une mise à distance de la technique [GAR 15].

\footnotetext{
${ }^{1}$ Une première version de ce texte a été présentée lors du séminaire Culture et Innovation, organisé à Lisbonne en décembre 2003 par

l'Institut franco-portugais.
} 
Prenons l'Europe. Son histoire matérielle et culturelle connait de profondes ruptures entre le XVI ${ }^{\mathrm{e}}$ et le $\mathrm{XVIII}^{\mathrm{e}}$ siècle. Citons, dans le domaine qui nous préoccupe, la première expansion mondiale au $\mathrm{XVI}^{\mathrm{e}}$ siècle, l'avènement de la science moderne au XVII ${ }^{\mathrm{e}}$ siècle, l'irrésistible développement du capitalisme industriel au XVIII ${ }^{\mathrm{e}}$ siècle. On s'attendrait à ce que de tels changements se soient effectués dans un bain de pensée exaltant le neuf, le progrès, qu'ils aient été accompagnés par une culture glorifiant l'innovation. Il serait exagéré de dire qu'il n'en fut rien. Les Théâtres de machines rédigés par les ingénieurs et publiés entre $\mathrm{XVI}^{\mathrm{e}}$ siècle et $\mathrm{XVIII}^{\mathrm{e}}$ siècle, louent la nouveauté et la mettent en avant [VER 04] ; les multiples traités techniques publiés au XVII ${ }^{\mathrm{e}}$ siècle insistent sur leur caractère novateur ; au XVIII ${ }^{\mathrm{e}}$ siècle, les Encyclopédistes plaident pour l'élargissement des cartes du savoir. Pourtant, l'innovation est unanimement décriée. Pire, jusqu'au XVIII ${ }^{\mathrm{e}}$ siècle, elle est redoutée. On observe à partir du XVI ${ }^{\mathrm{e}}$ siècle une prise de conscience autour de la pensée technique ; la technicité s'objective alors comme une manière singulière de maîtriser l'action et ses langages. Il n'en résulta aucune culture d'innovation.

\section{La technicisation des pratiques au $X V I^{e}$ siècle}

L'Europe du XVI ${ }^{\mathrm{e}}$ siècle initia une culture nouvelle de l'écrit technique. Elle œuvra à en fixer les règles, à en définir les spécificités. Le maitre-mot : «in artem redigere », que je traduirai par « conférer une langue écrite » aux pratiques et à l'action en général, avec ce que cela suppose de normes et de méthode [DUB 08]. Ce mouvement de pensée participait du mouvement de grammatisation qui saisit alors l'Europe intellectuelle [AUR 94]. L'idée générale de cette révolution intellectuelle dont nous restons aujourd'hui encore, et peut-être plus que jamais, tributaire : la compréhension du monde passe par la compréhension des langues qui s'en saisissent. Ce qui requiert de les objectiver en élaborant une sciencia, un savoir écrit, donc à en détecter les grammaires, à en définir les mots, les expressions, les expliquer, les comparer.

La pratique, terme qui ne désignait pas alors le savoir-faire des artisans mais l'agir nécessaire à la vie en société [CIF 01], était pensée comme un langage, une rhétorique qu'il était nécessaire de « réduire en art », comme le préconisait Cicéron. Les lettrés se donnèrent pour tâche d'en détecter la grammaire et de la publier. Avec cette nécessité en corollaire de définir une méthode spécifique pour rédiger au mieux ces écrits techniques.

L'avènement de cette culture technique d'un genre nouveau est lié au développement de l'imprimerie. Plus exactement, elle est liée à l'espoir que cette nouvelle technique souleva chez les intellectuels, à la manière dont elle les inspira. Leo Marx explique la disparition de l'optimisme technologique qui imprégnait la culture américaine du XIXe siècle aux années 1970, par la succession de désastres technologiques : Tchernobyl, Three Miles Islands, etc. [MAR 94]. Un mouvement strictement inverse s'est produit dans l'Europe du XVI ${ }^{\mathrm{e}}$ siècle. Un profond sentiment de perte imprégnait la culture occidentale depuis la fin du XIV $\mathrm{X}^{\mathrm{e}}$ siècle, après les ravages causés par la peste noire, qui se traduisait dans les milieux intellectuels par un sentiment de perte des savoirs. L'imprimerie leur parut le moyen de conjurer cette perte dans le futur. Contrairement aux manuscrits, en effet, l'imprimerie présentait cette caractéristique inédite d'assurer une transmission à l'identique des écrits, et une transmission qui, à l'époque, paraissait pouvoir s'inscrire dans la très longue durée, du simple fait, que tout écrit, pourvu qu'on en garde les supports initiaux, planches de bois ou un peu plus tard de cuivre, semblait reproductible à l'infini.

La réduction en art devint l'exercice majeur des experts placés, du fait de leur métier, en pédagogue et/ou formateur des princes et des élites : juristes, administrateurs, architectes, ingénieurs, mais aussi maître d'escrime, maitre à danser, avec dans tous les cas, le souci de créer, chacun dans son secteur, une «technè » au sens où l'entendait Platon, une culture technique partageable entre les praticiens et leurs clientèles, matérialisée à la fois par la qualité d'écriture des ouvrages, leur clarté, leur logique, leur souci d'être complet, et par les résultats, traités de gouvernement, méthodes historiques, normes 
sociales, bâtiments privés et publics, ordonnancements de jardins compréhensibles et appréciables par tous [BRI 02], [COU 96], [MAN 02], [PAU 12]. Célèbres pour l'esthétique de leurs gravures, et bien plus pertinents au plan technique qu'on ne l'a longtemps cru, les Théâtres de machines contribuèrent eux aussi au développement d'une culture technique partagée entre techniciens et usagers [THE 16]. Rédigés par des ingénieurs et des architectes, ils avaient pour objectif de montrer par le dessin des réalisations techniques, équipement militaire, équipement de jardin, de maison, équipement industriel, équipement énergétique, en place ou prototypes, et de les proposer comme modèle aux commanditaires, ou tout simplement aux amateurs et possibles clients, voire aux ingénieurs des générations à venir.

\section{Un nouveau régime de la technique, mais pas de culture d'innovation}

Francis Bacon théorisa la nécessité pour toute société immergée dans une compétition économique de disposer d'une culture technique et scientifique, débordant des savoirs des métiers. La Maison de Salomon, qui devait être le lieu de vie et de recherche des savants stipendiés par le pouvoir [RUE 16], s'avéra une source d'inspiration, pour ne pas dire un modèle d'action : la Royal Society of London for Improving Natural Knowledge reconnue officiellement à Londres en 1662 par Charles II [BRI 96], l'Académie royale des Sciences, officiellement fondée par Colbert en 1666 [MAZ 02] [DEL 17] s'en inspirèrent fortement. Pourtant, on ne trouvera aucune culture d'innovation chez Bacon, ni chez les savants et les gestionnaires qui s'inspirèrent de ses idées [GOD 15]. La culture qu'ils préconisent tous est une culture d'invention et d'expérimentation.

Francis Bacon exprime son sentiment sur le fait, signe au passage que la question se posait. Le sens commun considère aujourd'hui l'innovation comme une invention qui a réussi, l'invention étant la nouveauté en soi, et l'innovation, cette même nouveauté socialisée, c'est-à-dire intégrée économiquement. Mais au $\mathrm{XVI}^{\mathrm{e}}$ siècle, Bacon, comme tous les lettrés de son époque, maîtrise parfaitement le latin, la langue de partage et d'échange des savants européens. Pour lui, l'invention, du latin inventio, est littéralement, ce qu'on trouve, inventus, ce qu'on découvre, qui existe sans avoir été détecté, ou compris, ou repéré. L'innovation, du latin innovare, suppose d'introduire de la nouveauté, d'opérer un changement. Avec l'invention, on est dans le registre de l'amélioration, du perfectionnement, du progrès :

« Si l'utilité d'une unique invention particulière a tellement frappé les hommes qu'ils ont jugé supérieur à l'humanité celui qui a pu par un bienfait s'attacher tout le genre humain, combien il paraitra plus noble encore d'inventer ce par quoi toute autre chose peut être aisément inventée ! (Bacon, Novum Organum, 1986)

S'enthousiasme-t-il dans le Novum Organum. Avec l'innovation, on est dans le registre du changement radical, du risque sociétal. Le philosophe plaide pour l'invention, qui œuvre ad meliorem, pour le meilleur, dont il se recommande lui-même :

« Si en cela je me suis parfois écarté de ce qui est couramment reçu, ce fut dans l'intention de procéder in melius (pour le meilleur) et non in aliud (pour ce qui est autre), dans un esprit qui tend à l'amélioration et au développement non au changement et à la transformation... » (Bacon, Du progrès et de la promotion des savoirs 1605, 1991)

Il réitère le propos dans son essai sur l'innovation, : "Il serait bien que les hommes quand ils innovent suivent l'exemple du temps, qui lui, en effet, innove grandement, mais tranquillement et par degrés à peine perceptibles» (Bacon, De Augmentis scientarum, 2011). Il la perçoit plutôt positivement, quand il s'agit de méthode, négativement pour le reste en raison de son caractère brutal, en particulier lorsqu'elle concerne le politique. 
Jusqu'à la fin du XVIII siècle, il en fut ainsi : tandis que l'idée se répandait dans les élites sociales qu'il était nécessaire de se saisir des cultures professionnelles, des cultures de métier et de les transformer en cultures techniques ouvertes, partageables et perfectibles, nulle part ne se trouve l'idée qu'il faut développer l'innovation. Pour Diderot, l'invention est indispensable car elle élargit la carte du savoir, elle ouvre à l'agir de nouveaux chemins. Ce pourquoi, il en fait l'éloge, ce pourquoi aussi, il condamne les « funestes secrets » des Métiers, leur « routine », c'est-à-dire l'habitude prise de garder la complète propriété des savoir-faire, de la cacher et de n'en délivrer la connaissance qu'à un nombre limité d'apprentis, et avec elle la connaissance des possibles renouvellements.

Le philosophe plaide abondamment pour l'élargissement des connaissances, la mise à disposition publique du plus grand nombre de savoirs possibles. Cette idée, qui fonde l'Encyclopédie, fut aussi l'idée maîtresse de la législation sur les brevets, lors de la période révolutionnaire. On y retrouve une gestion habile de ce qui pourrait a priori s'opposer, savoir la propriété intellectuelle et la mise à disposition des connaissances : l'inventeur voit sa réputation garantie et les droits sur son invention protégés, un certain temps du moins, à condition qu'il en fasse publication, et donc qu'il la mette à disposition. Tandis que l'innovation, à l'instar de ce qu'écrivait Bacon au XVII siècle, demeure quelque chose de redoutable à penser, parce qu'elle débouche inévitablement sur le renouvellement des structures. Aussi bien lorsqu'on l'évoque, on la place dans le nulle part, dans un lieu imaginaire descriptible certes mais sans emprise sur le réel. L'invention s'inscrit dans la culture technique; l'innovation, elle, relève de l'utopie [GAR 05].

\section{Mais comment faisaient les entrepreneurs avant Schumpeter?}

On ne peut réduire le tissu économique d'avant le système fer/charbon/vapeur, à la protoindustrialisation. Ce type de business model, dominé par le capital commercial qui réduisait en soustraitance les artisans et les paysans-artisans, en leur faisant supporter l'investissement dans le capital fixe et l'acquisition des matières premières, - un business model que l'économie redécouvre aujourd'hui avec l'ubérisation- caractérisait le secteur textile principalement. L'industrie métallurgique européenne relevait d'autres modèles, soit valorisation du foncier par les propriétaires, soit constitution de compagnies actionnaires, soit encore blocs privés-publics, avec petites compagnies actionnaires et fonderie d'état.

La France du XVIII ${ }^{\mathrm{e}}$ siècle a connu de puissantes compagnies minéro-métallurgiques, en Alsace, en Lorraine, dans les Cévennes, le Lyonnais, les Pyrénées, dans les Alpes, et dans le Massif Armoricain. Ces compagnies étaient de grandes entités industrielles, dans un monde qui ne l'était pas. Dirigées par des ingénieurs, financées par des actionnaires, leur investissement initial comportait une part majeure de capital fixe. Il n'y a à la vérité aucune différence dans leur manière de pratiquer l'innovation et d'investir dans un matériel moderne, au nom de la recherche d'une plus grande efficacité et du profit, en adaptant des procédés métallurgiques aux nécessités de terrain, adoptant précocement de nouveaux procédés, machines à vapeur, fonte au coke, laminage à l'anglaise dans les années 1780 en Normandie [BEL 91] [BEL 92] [BEN 97]. Certaines innovèrent jusque dans leur mode de gestion, avec l'invention de la comptabilité technique dans les fonderies de Poullaouen, destiné à faire modèle pour les élèvesingénieurs des mines jusque dans les années 1860. Il s'agit bien d'innovations, c'est-à-dire de mise en œuvre de procédés totalement neufs (fonte au coke, comptabilité technique, laminage à l'anglaise), ou interprétation nouvelle de procédés anciens (réduction des métaux au four à réverbère). Mais ce fut sans développer une culture de l'innovation. Les entrepreneurs innovaient, sans éprouver le besoin d'objectiver leurs pratiques innovantes.

Mais ils comprirent vite qu'il leur manquait les cultures opératoires adéquates. Ces entrepreneurs et ingénieurs d'industrie n'eurent de cesse d'œuvrer à l'adoption, voire à l'élaboration de cultures techniques congruentes aux innovations techniques pour lesquelles ils optaient [GAR 02]. Beaucoup en firent l'expérience cuisante : qu'ils fassent venir des experts, y compris réputés, d'Allemagne ou 
d'Angleterre, ou qu'ils empruntent à ces pays des procédés, le résultat était le même : l'échec à court terme. Et cela pour une raison toujours revenue : en ces temps de savoirs pratiques liés à un complexe technique local et/ou régional, à sa langue, à ses cultures, à ses habitus techniques, à son contexte géologique, l'écart entre la culture technique de l'expert et celui du milieu dans lequel il était appelé à intervenir. Impossible pour l'expert confirmé venu d'Alsace pour faire fonctionner un four à coupellation dans les Cévennes, faute d'une connaissance adéquate des matières premières disponibles; impossible à l'ingénieur-directeur de Poullaouen de maintenir l'utilisation des cylindres en cuivre, pour les pompes assurant l'exhaure, faute de compétences techniques en alésage; impossible encore sur le même site, de maintenir en fonctionnement la machine de Newcomen faute d'une connaissance réciproque de la part du mécanicien et de l'ingénieur des spécificités de fonctionnement de la machine et d'organisation de ce milieu minier particulier qu'imposaient les gisements de plomb argentifère; échec de l'implantation au Creusot d'une usine sidérurgique dernier cri, etc [GAR 95] [BEL 11]. Les entrepreneurs, tout innovants qu'ils étaient, et parce qu'ils étaient innovants, soucieux d'adopter les procédés censés être les plus performants, découvrirent à leurs dépens qu'ils devaient élaborer, autour des objets nouveaux, un savoir d'appui, des cultures opératoires adéquates. En matière d'innovation, la mobilité des experts n'engendre pas en soi la co-activité. Il faut en complément disposer de milieux techniques ouverts à l'hybridation entre l'ancien et le neuf, ou, à défaut les susciter.

Force est de constater, pour conclure ce point, que l'histoire des entreprises pré-industrielles confirme deux aspects de la théorie C/K [LEM 14] : 1) concevoir un objet, un procédé ou un matériau n'est pas le connaître, loin s'en faut ; 2) le processus innovant suppose une conception et l'élaboration de connaissances permettant l'utilisation dans la durée du procédé. Dans le monde pré-industriel européen, la phase de constitution du domaine de connaissance n'est pas passée par la recherche d'une culture d'innovation, mais par la recherche de cultures opératoires mettant en jeu d'abord et peut-être surtout, le régime de la pratique, les savoirs informels sur l'objet, ce pourquoi d'ailleurs, spontanément, on fait venir des spécialistes. Leur échec à court terme fit émerger la question de la co-activité, donc l'élaboration d'une culture technique, ou technico-scientifique commune à l'ensemble des acteurs, entrepreneurs, ingénieurs, administrateurs et maîtres-ouvriers. Ce fut en Allemagne la raison de la multiplication des enseignements en technologie à partir des années 1750 et leur importation en France à la fin du XVIII ${ }^{\mathrm{e}}$ siècle, ce fut encore la raison fondatrice de l'Ecole des mineurs de Saint-Etienne dans le milieu des années 1810 et celle de son succès auprès de l'entrepreneuriat français [LAB 12] [GAR 04] [GAR 12].

\section{Une culture « tableau de bord » en complément des cultures opératoires}

La grande ambition de l'École des Mineurs de Saint-Étienne était de former des ingénieurs praticiens, aptes à contribuer à la modernisation de l'outil industriel à l'échelle du pays. Par chance, nous disposons des cours qui furent professés à l'orée des années 1830, au moment où professer l'industrie était en soi une innovation. On y découvre comment ces élèves-ingénieurs étaient formés à la modernité, et donc à l'innovation.

Premier constat, la nouveauté est largement présente, sous ses deux formes, théorique et pratique. Toutes les innovations des années 1820 sont présentes, le chemin de fer, la chaux hydraulique, les ponts suspendus, l'éclairage au gaz, la théorie analytique de la chaleur, la fonte au coke, les débats sur la nature de l'acier, la théorie géologique d'Elie de Beaumont sur le système des montagnes, les débats des géologues entre plutonisme et neptunisme, etc. Toutes les innovations, matérielles et intellectuelles, étaient présentées, analysées scientifiquement, décortiquées, mises à disposition, proposées à l'étude et à l'imaginaire technique. Mais, ce qui frappe, à la lecture des cours, c'est que rien n'est donné pour révolutionnaire ou innovant. Au contraire, tout est posé comme allant de soi, y compris les techniques les plus neuves et les plus disruptives, tout est comme aplani, normalisé par la lecture technico-scientifique qui en est faite. 
La fonte au coke par exemple, présentée dans le cours de métallurgie : combien étaient-ils de hautsfourneaux à fonctionner de la sorte à ce moment en France (ou à avoir fonctionné, car la crise de 18271829 avait été cruelle à cette jeune sidérurgie) ? A peine une dizaine. Or, tout de leur fonctionnement est énoncé comme allant de soi. L'introduction du coke dans le cours de métallurgie est presque anodine, ce qui ne veut pas dire, loin s'en faut, qu'elle est inexistante ou insuffisante. Bien au contraire, il imprègne de sa présence tout l'exposé, mais sur le registre de la normalité. Aucun chapitre spécifique ne lui est consacré, à la différence, par exemple, de l'acier. Le nouveau combustible est totalement intégré dans l'énoncé des procédés métallurgiques, à l'instar de ce qui se passe dans la définition préliminaire. Il est présent à chacune des phases, mais en toute discrétion. Le pragmatisme prime, une manière de libéralisme technique qui laissait à l'industriel le choix d'opter pour le meilleur des combustibles en fonction du temps dont il dispose, du lieu et des conditions dans lequel il se trouve, en fonction aussi du produit qu'il veut obtenir. L'idée dominante est celle de présenter, de proposer, non d'imposer, y compris au nom du progrès. Le caractère disruptif de la fonte au coke, au plan économique, était connu, en effet. Elle requérait en effet un lien commercial et institutionnel avec les compagnies minières, là où la fonte au coke se contentait d'un lien avec le foncier (la propriété des bois). En d'autres termes, la sidérurgie au bois était le fait de propriétaires fonciers, mettant leurs fourneaux en régie à des maîtres fondeurs, la sidérurgie au coke impliquait le capitalisme industriel. Fait notable, une part du cours de métallurgie était consacré à l'économie. A l'image du coke, l'innovation disparaît de toutes les manières, alors qu'elle est partout présente. Il n'y a dans les énoncés, aucune différence de traitement entre les procédés traditionnels et les procédés nouveaux. Tout contribue à la normalisation du produit innovant, dont on efface autant que faire se peut l'inquiétante étrangeté, que l'on banalise. L'innovation n'a de valeur qu'à condition de disparaître dans le paysage technique, à condition qu'elle s'y intègre, qu'elle ne le dérange pas, et cela jusque dans l'enseignement qui s'y rapporte.

Second constat, l'énoncé professionnel mobilise d'autres modes d'acceptation : la narrativité, cette mise en discours, presque une mise en scène, que vient renforcer à certain moment, l'usage du registre esthétique, pour ne pas dire du registre héroïque. Ainsi le cours de géologie, à partir de 1828 . L'ingénieur Fénéon, son auteur y incorpore ce qui se fait de mieux à ce moment : Fourrier, Cordier, Elie de Beaumont, Brongniart. Il se met en scène, visitant les Alpes en compagnie des deux grands savants, Elie de Beaumont et Léopold de Buch. Werner est cité et quelque chose de l'esprit d'aventure souffle indubitablement lorsqu'il décrit les Alpes et informe son auditoire sur les derniers états de la science géologique. Il met en scène l'hétéroclite naturel :

«Fossiles des grandes alluvions : les grandes alluvions contiennent : deux Crocodiles, un Tryonis, les ossements d'un Dauphin voisin de notre Epaulard, une Baleine analogue aux Rorquals et un genre nouveau très rapproché des Cachalots, et qui se compose de trois espèces. Avec eux des Pachydermes gigantesques, des Eléphants, des Rhinocéros, des Hippopotames, des Tapirs, un Sanglier, un grand nombre de très petits chevaux et quelques ruminants ».

L'ambiance du cours avoisine le sublime montagneux : on y parle de «prodigieuses abondances », de matériaux «charriés », "arrachés »; de "commotion violente », de " déchirement», de " dépouilles», de "révolutions nombreuses et subites», de " couches culbutées, contournées », de «masses arrachées, violemment déchirées». (Laboulais, La Maison des mines: la genèse révolutionnaire d'un corps d'ingénieurs civils, 2012).

D'aucuns y verront la marque du catastrophisme de Cuvier, bien que le grand homme ne soit pas mentionné. J'en retiens quant à moi, que ce discours fortement métaphorique donnait sens à l'histoire, celle des roches mais aussi celles des hommes. Tel, en effet, le cours théorisait crise et rupture en les incluant dans l'évolution. Tel encore, il affaiblissait l'impact de la disparition et du renouvellement en introduisant le recul historique. Fondamentalement quelle était la leçon donnée ? Dans le cadre d'une science qui posait «l'ordre des phénomènes pour arriver si possible à leur cause réelle » d'une science 
qui disposait de ces faits tangibles qu'étaient les fossiles, l'élève apprenait que la terre était passée par une série de vicissitudes avant de parvenir en son état de tranquillité ; que le «globe a éprouvé des révolutions nombreuses et subites » dont la dernière « a totalement anéanti la race des mastodontes ». ${ }^{2}$

Dans cet apprentissage conjoint de la nouveauté et de la manière de l'introduire, le registre scientifique donnait sens. Il constituait un allié précieux parce que, parfaitement maîtrisé par l'ingénieur professeur, il engendrait l'évidence. En d'autres termes, en complément de l'indispensable culture technique, l'école distillait une culture «tableau de bord » pour reprendre l'expression d'Owen Barley [BAR 88]. Les élèves étaient formés à chacune des cultures techniques propres à chacune des spécialités qu'ils étaient amenés à pratiquer ou à côtoyer dans leur vie professionnelle. Une culture générale était distillée, en complément de ces approches strictement opératoires, où se mêlaient, dans la même approche pragmatique, esthétique et narrative, une philosophie de la rationalité scientifique facteur de progrès et la vulgarisation des méthodes et des pratiques scientifiques. Cette culture «tableau de bord», qui manquait à l'entreprise pré-industrielle, constituait un habitus partagé par l'ensemble des élèves sortant de l'école, quelque fût ensuite leur métier, directeur d'usine, ingénieur praticien, maître mineur ou garde-mines, selon leurs origines et/ou leur résultats.

\section{Quand la culture « tableau de bord » devient culture d'innovation}

Allons vers d'autres ingénieurs d'industrie, dans les années 1890, cette fois, ces ingénieurs, Gadzarts et Centraliens majoritairement, qui publient abondamment dans la revue La Nature. Là, incontestablement apparaît une culture de l'innovation, mais dans un registre autre, celui de la vulgarisation scientifique et technique. L'innovation y est enfin désignée nommément et envisagée jusque dans sa capacité à détruire. Et c'est pour faire face à cette capacité de destruction que le journal propose des procédures intellectuelles, des garde-fous. Deux niveaux de discours se distinguent. D'une part, le journal met en scène, vulgarise la culture technique propre à chaque domaine de l'ingénierie industrielle, en donnant corps à la figure de l'amateur expert, au passage. D'autre part, à un plan général, ce qui se fabrique dans La Nature c'est une topique de pensée globale et cohérente autour des concepts nouveaux, tant matériels qu'immatériels, un sens commun de l'innovation. Et, fait notable, cette topique de pensée incorpore, dans le même régime d'appropriation, les acteurs immédiats de la technique, les usagers, et au-delà encore, les spectateurs, le public, ce qui n'était pas le cas auparavant.

Cette nouvelle topique de pensée, véritable lingua franca de l'innovation que j'ai étudiée en me focalisant sur les objets de déplacement automobile, voitures, tricycles, camions, trottoirs roulants, escaliers roulants, tous présents et décrits dans la décennie 1890-1900, adopte les contours suivants :

$\left.1^{\circ}\right)$ L'innovation prend clairement son origine dans deux rêves, celui de l'ingénieur et celui du consommateur. Il y a là quelque chose du " vous l'avez rêvé, nous l'avons fait », que l'on rencontre régulièrement dans les slogans publicitaires. Le journal évoque le rêve de l'usager. Ainsi à propos des véhicules automobiles : « Sans réaliser le rêve du touriste ou du commerçant, la voiture à gazoline est dès à présent entrée dans la pratique » (Hospitalier, Voitures automobiles. Les lauréats du concours du Petit Journal, 1894) ; ou encore, sur un autre registre :

«Les médecins, les hommes d'affaire (...) applaudiront aux efforts des constructeurs qui travaillent pour eux et leur préparent des voitures avec lesquelles il ne sera pas possible de rouler à plus de vingt kilomètres par heure, mais qui permettront de monter toutes les côtes et de se transporter économiquement » (E. Hospitalier, La Nature, 1898).

Partir du rêve a une fonction : cela fixe le portrait de la machine idéale, donne le but à atteindre, et dans le même mouvement, donne ses limites à sa concrétisation. Le rêve fait office d'horizon de pensée.

\footnotetext{
${ }^{2}$ Cf. Fénéon, Cours de géologie, Ecole Supérieure des Mines de Paris, non daté.
} 
$2^{\circ}$ ) Il existe deux sortes d'innovations. La première, l'innovation absolue, ne ressemble à rien de connu. Cette catégorie suscite méfiance et scepticisme. Ainsi, le moteur Diesel à la fin des années 1890. Le premier temps est celui de la réticence, voire de la moquerie :

«Empressons-nous de signaler cette invention pendant que les périodiques d'outre-Rhin la placent au Capitole. Quelques semaines de retard nous exposeraient peut-être à la trouver au bas de la roche Tarpéienne »(J.B., Le moteur Diesel, 1897).

Le deuxième temps est celui de l'acceptation, sous condition toutefois d'introduction de référents. Pour le moteur Diesel, cela vient vite: en l'espace d'une semaine, le ton est rectifié parce qu'une démonstration de cette machine a été effectuée devant une communauté d'ingénieurs. C'est du sérieux donc, la communauté technicienne en fait foi :

« Le moteur d'essai a été construit à Augsbourg et présenté le 27 avril ; le 16 juin 1897, il était décrit devant la Société des ingénieurs de Cassel. La figure 1 ci-jointe donne une vue extérieure du moteur.... En résumé, le moteur Diesel semble présenter de grands progrès sur les moteurs actuels, et il n'est encore qu'à ses débuts ; il est donc susceptible de grands perfectionnements » (Laffargue, Le moteur Diesel à combustion intérieure, 1897).

La deuxième sorte d'innovation est celle qui part d'un objet existant et le réaménage, le ré-agence. L'acceptation dans ce cas va de soi. Les exemples sont nombreux parmi lesquels nous pouvons choisir celui du « Tricycle automobile», qui est :

«...un petit automobile à trois roues, rasant le sol, (que l'on voit) traverser rapidement les rues et les boulevards, évoluer avec facilité au milieu des encombrements de voitures et disparaitre dans la cohue des passants sans autres bruit que celui de l'échappement des gaz brûlés » (Baudry de Saunier, Le tricycle automobile Bollée, 1896).

Voilà, pour le journal, une «bonne innovation », c'est-à-dire une innovation qui correspond pleinement à ce que l'ingénieur sait, peut et doit faire, améliorer ce qui existe et non créer :

« le tricycle automobile en question ne renferme d'innovations en aucune de ses parties, mais qu'il réunit avec adresse tous les progrès que l'industrie des voitures automobiles a réalisés aujourd'hui... M. Bollée a eu là une conception bien nette, suivie d'une très habile exécution, de la voiturette automobile construite selon les principes du cyclisme, et pour laquelle une si énorme clientèle est déjà prête» (L. Baudry de Saunier, Le tricycle automobile Bollée, 1896).

On notera d'une part le renversement de sens qui s'est effectué entre « invention » et innovation »; la nouveauté absolue qui était « innovation » à l'époque moderne est devenue « invention». Mais, audelà du changement de sens, il y a une permanence : l'innovation absolue fait peur. La réaction en deux temps après l'annonce de la mise au point du moteur Diesel montre qu'à l'orée du $\mathrm{XX}^{\mathrm{e}}$ comme au $\mathrm{XVI}^{\mathrm{e}}$ siècle, ce qui est redouté, c'est le changement brutal des normes.

$3^{\circ}$ ) Enfin, et cela est notable, les ingénieurs-rédacteurs de la Nature ont parfaitement compris que l'innovation élevait l'entropie du système. C'est en tout cas l'analyse qu'ils en font. Et ce qu'ils théorisent là, n'est rien moins que la création destructrice. Pour eux, l'innovation crée une rupture ; elle oblige à réaménager sans cesse le système, à déployer sans cesse de nouvelles techniques. Il y a dans La Nature cette conscience nette que la société change, que son environnement technique est aussi en plein changement, et que cela fait courir un risque à la société ! L'innovation accroît la responsabilité sociale de l'ingénieur, à qui il incombe de suivre ces changements, d'abaisser l'entropie. Le perfectionnement quotidien des appareils par les ingénieurs, dans le dessein de les simplifier est l'un des thèmes récurrents du journal. L'inachèvement en quelque sorte protège du délitement. 
Le rêve, le risque de rupture, l'endiguement par la capacité du génie industriel à perfectionner les nouveaux concepts et objet : tels sont les contours d'une topique de pensée socialise qui socialise de fait la nouveauté industrielle, à un moment, ne l'oublions pas, où le monde industriel défraye la chronique sociale. La crise économique bat son plein, le mouvement ouvrier est puissant, le marxisme a le vent en poupe. En regard, le génie industriel, lui aussi touché par le chômage, travaille à " produire sa réalité, de façon progressive et collective, à travers un travail constant de réécriture de lui-même par lui-même », pour paraphraser la définition que fait Antoine Hennion de l'innovation en entreprise [HEN 03]. On est en présence du bouillon de culture de la société industrielle de la fin du XIXe siècle, à partir de quoi s'est constituée la pensée des économistes et sociologiques de l'innovation, J. A. Schumpeter et W. F. Ogburn au premier chef [GOD 10].

\section{Pour conclure : que signifie l'objectivation d'une culture d'innovation au tournant des $\mathrm{XIX}^{\mathrm{e}}$ - $\mathrm{XX}^{\mathrm{e}}$ siècle?}

L'innovation, qui comprend la capacité humaine à user de nouvelles formes matérielles, de nouvelles lignées techniques et de nouvelles formes symboliques, l'innovation donc, est de toutes les époques et de toutes les sociétés et groupes humains. Elle trouve son fondement dans la technicité, cette aptitude qu'ont un grand nombre d'êtres vivants à transformer leur milieu de vie, à se doter d'un capital technique en élaborant des savoir-faire normés, transmissibles et transférables.

La singularité de l'espèce humaine est d'avoir géré son aptitude à la technicité en l'individualisant, d'en avoir fait une pratique objectivée. "La technicité, écrit G. Simondon, est le degré de concrétisation de l'objet», c'est-à-dire le degré de concrétisation de la topique de pensée forme/matière/fonctionnement. L'imagination technique se déploie grâce à " une sensibilité particulière à la technicité des élements; c'est cette sensibilité à la technicité qui permet la découverte des assemblages possibles ", et conduit donc, in fine, à l'innovation. Cela s'est fait au moyen d'un régime spécifique d'appropriation du fait technique, distinct des modes de structuration des technicités animales, structuré par l'oralité et tout ce qui s'y rapporte, usage des sons, avec un usage intensif de l'interprétation symbolique, métaphorique du monde [SIM 89, 92, 12]. L'homme n'a pas d'abord inventé l'écrit; il a d'abord entremêlé ses deux capacités de technicité et d'imaginaire, pour normer et transmettre en toute conscience ses savoir-faire, en développant de multiples symbolismes techniques. Les cultures de métiers se sont structurées autour de l'oralité et de la capacité humaine à normer et à transmettre par le symbolise. Nous appellerons ces cultures de métiers, des «cultures techniques originelles ». Comme toute culture technique, elles comportent un vocabulaire et/ou une langue de métier, le technolecte, et d'un habitus technique, ces ensembles de gestes, rites dont la pratique autorise et/ou détermine l'efficacité, dans le cadre d'une culture donnée. Elles continuent d'exister, y compris dans les milieux de travail les plus sophistiquées, quoiqu'elles demeurent incomprises et sousestimées. Elles étayent la capacité humaine à innover, sans toutefois l'objectiver, sans objectiver non plus, le fait technique en soi. Ce qu'elles objectivent, c'est la technicité, la capacité humaine à imaginer la relation forme/matière/fonctionnement, en la concrétisant dans l'objet ou dans l'outil, et en la mettant en scène par le symbole, pour en assurer la transmission.

L'élaboration du régime de la technique traduit un changement radical dans l'appréhension du fait technique. Ce changement s'est effectué en lien avec l'organisation de chantiers et/ou d'ateliers complexes, selon toute vraisemblance, parce qui exigeaient une gestion élargie et rigoureuse des matériaux et l'orchestration de multiples savoir-faire. Ainsi, les énormes chantiers de construction des IVe et IIIe millénaire avant notre ère. Ainsi, les exploitations minières de grande envergure de l'époque romaine. Ainsi la construction du gigantesque système hydraulique de Dujiangyan, dans le Sichuan au $3^{\text {ème }}$ siècle de notre ère, de la Grande muraille et du Grand canal, trois siècles plus tard. L'invention de l'écrit a rendu possible son expression pleine et entière en favorisant l'objectivation des normes, la mise à distance des savoir-faire et l'organisation de nouvelles modalités de transmission. Cette évolution dans les modes d'appropriation du fait technique, a émergé localement, régionalement, pour 
s'atténuer ensuite, voire disparaître de longs siècles. Certainement, il accompagne la mise sur pied de l'administration chinoise, alors que s'invente l'imprimerie, dans les premiers siècles de notre ère. Il entre pleinement et durablement dans l'histoire européenne à partir du XVIe siècle, en lien là aussi avec l'imprimerie. L'analyse de ce moment historique de l'histoire occidentale permet de faire trois observations :

1) cette objectivation est prise en charge par une catégorie socio-professionnelle qui se développe alors avec force : l'ingénieur, dont le champ d'action est tantôt militaire (fortifications et engins militaires), tantôt civil (machinisme hydraulique, outillage industriel) ;

2) le nouveau régime de la technique adopte les contours de la culture générale des élites savantes, en l'occurrence : mise par écrit, grammatisation des pratiques, qui lui sert d'étayage, de topique de pensée. Traités et manuels techniques deviennent des outils indispensables d'élaboration, de diffusion et de transmission des connaissances techniques et/ou des outils de gestion des savoirfaire. L'objectif est de parvenir à une universalité de l'expression technique. L'énoncé technique abandonne le symbolisme, trop lié aux cultures locales, au profit de vocabulaires «neutres » véhiculant des termes précis. L'école devient un lieu majeur de la transmission des connaissances techniques. La culture technique des catégories socio-professionnelles concernées, langue et habitus, s'éloigne du régime de la pratique, refoulé dans les arcanes de l'intuition, et s'enchâsse dans le mode de pensée scientifique d'autant plus aidément que le régime de la technique a contribué à l'élaborer, aux XVII ${ }^{\mathrm{e}}$ et XVIII ${ }^{\mathrm{e}}$ siècles. L'efficacité technique s'est alors mathématisée.

3) cette nouvelle manière de concevoir le fait technique, fut en soi une innovation. Mais ce caractère innovant n'a pas été objectivé en tant que tel. Ce qui a été objectivé, c'est le rôle social de la technique. Et cette objectivation a donné sens à la notion de progrès. Par deux fois au moins, philosophes et savants se sont appuyés sur le progrès technique pour démontrer la capacité humaine à aller de l'avant, à faire mieux que les anciens : au début du XVI siècle, avec Francis Bacon, à la fin du XVII siècle, lors de la Querelle des Anciens et des Modernes. Pour autant, ce tournant paradigmatique dans l'histoire de la pensée européenne, n'a pas donné naissance à une culture de l'innovation. Mais son développement a obligé les divers acteurs de la technique à repenser la question de la co-activité. Cela a débouché sur l'élaboration à partir du $\mathrm{XVIII}^{\mathrm{e}}$ siècle d'une culture technico-scientifique neuve, partageable entre ingénieurs, entrepreneurs, administrateurs et maitres-ouvriers, une " dashboard culture », véritable topique de la pensée ingénierale jusqu'à la fin du XIX ${ }^{\mathrm{e}}$ siècle qui s'est établie autour de trois pôles : la technique (celle du régime de la technique, pas celle du régime de la pratique), le progrès, la science. La culture technique d'un ingénieur s'étoffe d'autant. Elle comprend désormais: la vieille culture de la pratique, informelle, utilisée pour « intuiter », et pour accomplir les basses œuvres de la réalité technique; la culture technique stricto sensu, culture de l'efficacité de métier, culture scolaire parfois, livresque et scientifique toujours, autour de laquelle s'organise la conception règlée [LEM 14]; la « dashboard culture » enfin, saint-simonienne par exemple en France, support de l'idéologie industrialiste, partagée par la majorité des acteurs du secteur, entrepreneurs, ingénieurs, contremaîtres, et bien souvent jusqu' aux ouvriers.

C'est cette « dashboard culture » qui va être le support de la culture d'innovation, qui émerge dans les pays industriels, entre la fin du $\mathrm{XIX}^{\mathrm{e}}$ et le début du $\mathrm{XX}^{\mathrm{e}}$ siècle, avec l'électricité, le pétrole, l'aluminium, le téléphone, l'automobile, l'avion, tous ces matériaux et objets techniques que le génie industriel mobilise, invente et/ou déploie au tournant des XIX ${ }^{\mathrm{e}}$ et $\mathrm{XX}^{\mathrm{e}}$ siècles, dans une phase de forte perturbation économique. Alors, le potentiel innovant de la technique est objectivé, et traité pour tel. Le caractère disruptif du nouvel environnement technique est nettement perçu, en effet. En 1910, le Manifeste technique de la peinture futuriste exaltait les mérites de la destruction créatrice de la nouvelle civilisation industrielle. Au même moment, le génie industriel, concepteur des objets techniques exaltés par les Futuristes, travaillait à rendre socialement acceptable des créations 
conceptuelles dont il mesurait la potentialité destructrice. La nécessité nouvelle d'introduire socialement l'innovation, et donc d'inventer une culture adéquate fait écho à un changement de business-model dans le monde industriel : les grandes entreprises pré-industrielles évoluaient dans des marchés segmentés, à la dynamique incertaine ; les grandes entreprises de la première industrialisation avaient pour clients privilégiés, les autres grandes compagnies industrielles, les marchés publics, civils et militaires; les objets techniques mis au point dans le cadre du système pétrole/électricité/alliages, s'adressent, eux, d'abord au public, en tant que potentiel acquéreur ou usager. Une nouvelle topique de pensée émerge chez les plus innovants, ou tout simplement les plus clairvoyants, qui s'architecture autour de trois pôles, le rêve, la rupture et le management scientifique, qui fonde la culture d'innovation à l'occidentale. Elle est désormais partagée par tous. Mais, dans le monde des concepteurs, elle est devenue culture technique, CAO et Big data aidant [GAR 16]. Un nouveau mode d'appropriation du fait technique en est résulté, le régime de la technologie.

\section{Bibliographie}

[ARE 94] ARENDT H., Condition de l'homme moderne (The Human condition), trad. G. FRADIER, Calmann-Lévy, Paris, 1994.

[AUR 94] AUROUX S., La révolution technologique de la grammatisation. Introduction à l'histoire des sciences du langage, Mardaga, Liège, 1994.

[BAC 86] BACON F., Novum Organum, édité par M. MALHERBE et J.-M. Pousseur, PUF, Paris, 1986.

BAC 91] BACON F., Du progrès et de la promotion des savoirs (1605), édité par M. LE DeEUFF, Gallimard, Paris, 1991.

[BAC 11] BACON F., De Augmentis scientarum, livre VI, antithèse XL; Essai XXIV, « Of innovations », In J.-M. RoBertson (dir.), The Philosophical Works of Francis Bacon, Abingdon, New York, Routledge, 2011.

[BAR 88] BARFIELD O., Saving the Appearances: A Study in Idolatry, Wesleyan University Press, Middletown, CT, (1957) 1988.

[BEL 91] Belhoste J-F., La Métallurgie normande: XII ${ }^{e}$-XVII ${ }^{e}$ siècles : la révolution du haut-fourneau, Association histoire et patrimoine industriels de Basse-Normandie, Caen, 1991.

[BEL 92] Belhoste J-F., Fonte, fer, acier: Rhône-Alpes, $X V^{e}$-début XX $X^{e}$ siècle, ADIRA Rhône-Alpes, Lyon, 1992.

[BEL 11] BeLHOSTE J-F., « La question de l'acier en France au XVIII 'e siècle : l'histoire d'un rendez-vous manqué entre science et industrie », In Ph. DILlmanN, L. PEREZ, C. SERNA (dir.), L'acier en Europe avant Bessemer, CNRSUniversité Toulouse-Le Mirail, Toulouse, p. 129-145, 2011.

[BEN 97] BENOIT P., La mine de Pampailly, XV $-X V I I I^{e}$ siècles, Brussieu-Rhône, SRA Rhône-Alpes, Lyon, 1997.

[BRI 96] BRIOIST P., «Les origines de la Société royale de Londres », La science à l'époque moderne. Actes du colloque de 1996, Bulletin de l'association des historiens modernistes des universités, $\mathrm{n}^{\circ} 21$, Paris, Presses de 1’U. Paris-Sorbonne, p. 91-121, 1996.

[BRI 02] BRIOIST P., «L'escrime à l'âge de raison », In P. BRIOIST, H. Drevillon, P. SERnA (dir.), Croiser le fer. Violence et culture de l'épée dans la France moderne (XVI'-XVIII siècle), Champ Vallon, Seyssel, p. 133-197, 2002.

[CIF 01] Cifoletti G., «L'utile de l'entendement et l'utile de l'action: Discussion sur l'utilité des mathématiques au $\mathrm{XVI}^{\mathrm{e}}$ siècle », Revue de Synthèse, t. 122, n 2-4, p. 503-520, 2001.

[COU 96] CouzINET M.D., Histoire et méthode à la Renaissance. Une lecture de la Methodus de Jean Bodin, Vrin, Paris, 1996.

[DEL 17] DelaunAy B., L’Académie royale des Sciences et la technique, XVII'-XVIII siècle, H. Champion, Paris, 2017.

[DUB 08] Dubourg GLATIGNy P., VÉRIN H., Réduire en art : la technologie de la Renaissance aux Lumières, Éditions de la Maison des sciences de l'homme, Paris, 2008.

[GAR 02] GARÇON A.F., HILAIRE-PÉREZ L., « Open Technique between Community and Individuality in EighteenthCentury France », In F. DE GOEY, J. W. VELUWENKAMP (dir.), Entrepreneurs and Institutions in Europe and Asia, 15002000, Aksant, Amsterdam, p. 237-256, 2002. 
[GAR 03] GARÇON A.F., "Comment retracer historiquement les chemins de la novation », In L. HILAIRE-PEREZ, A.F. GARÇON (dir.), Les chemins de la nouveauté. Innover, inventer au regard de l'histoire, éditions du CTHS, Paris, p. 443470, 2003.

[GAR 04] GARÇON A.F., Entre l'État et l'usine: l'École des mines de Saint-Étienne au XIXe siècle, PUR, Rennes 2004.

[GAR 05] GARÇON A.F., "Les techniques et l'imaginaire Une question incontournable pour l'historien », Hypothèses: Travaux de l'École Doctorale d'histoire de l'Université Paris I Panthéon-Sorbonne, n51, p. 221-228, 2005.

[GAR 12] GARÇON A.F., «The Three States of Technology. An Historical Approach to a Thought Regime, XVI ${ }^{\text {th }}-$ XX $^{\text {th }}$ Centuries », In M. FAUCheuX, J. Forest (dir.), New Elements of Technology, Presses de l'UTBM, Belfort, p. 11-26, 2012.

[GAR 15] GARÇON A.F., "Des modes d'existence du geste technique », e-Phaïstos. Revue d'histoire des techniques/Journal of History of Technology, p. 84-92, 2015.

[GAR 16] GARÇON A.F., "Quand l'automation percute l'agir opératoire (et réciproquement) : une autre lecture du Génie industriel... », In S. BenOIT ET A. Michel (dir.), Le monde du génie industriel au XX $X^{e}$ siècle. Autour de Pierre Bézier et des machines-outils, Presses de l'UTBM, Belfort, p. 33-43, 2016.

[GOD 10] GodIN B., « Innovation Without the Word: William F. Ogburn's Contribution to the Study of Technological Innovation », Minerva, vol. 48, n 3, p. 277-307, 2010.

[GOD 15] GodIN B., Innovation Contested: The Idea of Innovation Over the Centuries, Routledge, London, 2015.

[HEN 03] HENNION A., "L'innovation comme écriture de l'entreprise. Récits d'innovation au sein d'une entreprise de services », In P. Mustar, H. PENAN (dir.), Encyclopédie de l'innovation, Economica, Paris, p. 131-152, 2003.

[LAB 12] LABOUlaIS I., La Maison des mines: la genèse révolutionnaire d'un corps d'ingénieurs civils (1794-1814), PUR, Rennes, 2012.

[LEM 14] Le Masson P., Weil B., Hatchuel A., Théorie, méthodes et organisations de la conception, Presses des Mines, Paris, 2014.

[MAN 02] MANDOSIO J.M., « Méthodes et fonctions de la classification des Sciences et des Arts (XV'-XVII ${ }^{\mathrm{e}}$ Siècles) 》, Nouvelle Revue du XVI siècle, vol. 20, n 1, p. 19-30, 2002.

[MAR 94] MARX L., "The Idea of "Technology" and Postmodern Pessimism », In M. R. SMITH, L. MarX (dir.), Does Technology Drive History?: The Dilemma of Technological Determinism, p. 237-257, 1994.

[MAZ 02] MAZAURIC S., « Des académies de l'âge baroque à l'Académie royale des Sciences », In Ch. DEMEULENAEREDOUYere, E. Brian (dir.), Règlement, usages et science dans la France de l'absolutisme, Editions Technique \& Doc, Paris, 2002.

[PAU 12] PAUWELS Y., «L'architecture de la Renaissance entre ratio mathématique et ars rhétorique », Seizième Siècle, n8, p. 19-27, 2012.

[RUE 16] RUELLET A., « La maison de Salomon : contribution à l'histoire du patronage scientifique et technique. France et Angleterre, ca.1600-ca.1660 », e-Phaïstos. Revue d'histoire des techniques/Journal of History of Technology, vol. V, n 1, p. 10-15, avril 2016.

[SIM 12] Simondon G., Du mode d'existence des objets techniques, Aubier, Paris (1958) 2012.

[THE 16] THEBAUD-SORGER M., «Les publics des sciences et des techniques : médiations, réceptions », In L. HILAIREPEREZ, F. Simon, M. THEBAUd-SORGER (dir.), L'Europe des sciences et des techniques. Un dialogue des savoirs, $X V^{e}$ XVIII siècle, PUR, Rennes, p. 333-353, 2016.

[VER 04] VÉRIN H., DOLZA L., «Figurer la mécanique : l'énigme des théâtres de machines de la Renaissance», Revue d'histoire moderne et contemporaine, 2004/2, n'51-2, p. 7-37. 